Optimization of synthesis process of thermally-responsive poly-n-isopropylacrylamide nanoparticles for controlled release of antimicrobial hydrophobic compounds

This content has been downloaded from IOPscience. Please scroll down to see the full text. 2014 Mater. Res. Express 1045404

(http://iopscience.iop.org/2053-1591/1/4/045404)

View the table of contents for this issue, or go to the journal homepage for more

Download details:

IP Address: 128.194.202.184

This content was downloaded on 30/10/2014 at 13:57

Please note that terms and conditions apply. 


\title{
Optimization of synthesis process of thermally- responsive poly-n-isopropylacrylamide nanoparticles for controlled release of antimicrobial hydrophobic compounds
}

\author{
Laura E Hill and Carmen L Gomes \\ Department of Biological \& Agricultural Engineering, Texas A\&M University, College Station, \\ TX-77843-2117, USA \\ E-mail: carmen@tamu.edu
}

Received 29 June 2014, revised 8 October 2014

Accepted for publication 16 October 2014

Published 29 October 2014

Materials Research Express 1 (2014) 045404

doi:10.1088/2053-1591/1/4/045404

\begin{abstract}
The goal of this study was to develop an effective method to synthesize poly-nisopropylacrylamide (PNIPAAM) nanoparticles with entrapped cinnamon bark extract $(\mathrm{CBE})$ to improve its delivery to foodborne pathogens and control its release with temperature stimuli. CBE was used as a model for hydrophobic natural antimicrobials. A top-down procedure using crosslinked PNIPAAM was compared to a bottom-up procedure using NIPAAM monomer. Both processes relied on self-assembly of the molecules into micelles around the CBE at $40{ }^{\circ} \mathrm{C}$. Processing conditions were compared including homogenization time of the polymer, hydration time prior to homogenization, lyophilization, and the effect of particle ultrafiltration. The top-down versus bottom-up synthesis methods yielded particles with significantly different characteristics, especially their release profiles and antimicrobial activities. The synthesis methods affected particle size, with the bottom-up procedure resulting in smaller $(P<0.05)$ diameters than the top-down procedure. The controlled release profile of CBE from nanoparticles was dependent on the release media temperature. A faster, burst release was observed at $40^{\circ} \mathrm{C}$ and a slower, more sustained release was observed at lower temperatures. PNIPAAM particles containing CBE were analyzed for their antimicrobial activity against Salmonella enterica serovar Typhimurium LT2 and Listeria monocytogenes Scott A. The PNIPAAM particles synthesized via the top-down procedure had a much faster release, which led to a greater $(P<0.05)$ antimicrobial activity. Both of the top-down nanoparticles performed similarly, therefore the $7 \mathrm{~min}$ homogenization time nanoparticles would be the best for this application, as the process time is shorter and little improvement was seen by using a slightly longer homogenization.
\end{abstract}


Keywords: stimuli-responsive, synthesis optimization, hydrophobic antimicrobial, nano-encapsulation, antimicrobial activity, bottom-up and top-down processes

\section{Introduction}

Poly(N-isopropylacrylamide) (PNIPAAM) is a temperature-responsive polymer with a lower critical solution temperature (LCST) that ranges from $30^{\circ} \mathrm{C}$ to $35^{\circ} \mathrm{C}$ [1]. PNIPAAM possesses both hydrophilic and hydrophobic groups in the polymer chain that result in its unique LCST behavior [2]. At temperatures below the LCST, hydrogen-bonding interactions between water and the hydrophilic groups of the polymer lead to a swollen hydrogel state. Once temperatures are increased above the LCST, there is a weakening of the polymer-water hydrogen bonds and a strengthening of polymer to polymer interactions among the hydrophobic groups [3]. When this polymer contraction occurs, it forces out some of the active material entrapped within the polymer matrix, creating a burst release at the transition temperature [4]. The temperature range for PNIPAAM's LCST is similar to the optimal temperature range for microbial growth for several foodborne pathogens of interest. This temperature-responsive polymer could provide the ability to entrap antimicrobial material within PNIPAAM particles and then trigger its release when foods are stored at temperatures ideal for microbial growth. A temperature-triggered release of antimicrobial could reduce the incidence of foodborne pathogen related illnesses that occur as a result of improper food handling or storage.

PNIPAAM is one of the most commonly studied temperature-responsive polymers for drug delivery applications, but there are many different methods described to form PNIPAAM nanoparticles, primarily a top-down procedure and bottom-up procedure [5-7]. Currently, there is no consensus on the best method to synthesize PNIPAAM nanoparticles for controlled release applications, and no studies have compared the characteristics and performance of nanoparticles synthesized via different processes. This study sought to determine the best nanoparticle synthesis method and optimize the nanoparticle production for antimicrobial controlled release applications and to determine what stages of the synthesis procedure had an important impact on the final nanoparticles.

Essential oils are naturally occurring antimicrobial compounds extracted from herbs and spices [8]. Spice essential oils have shown enhanced antimicrobial activity compared to their corresponding isolated active compounds due to the presence of several different active compounds working synergistically to inhibit microorganisms [9, 10]. Cinnamon bark extract (CBE) contains three active compounds that inhibit bacterial growth through different mechanisms of action [11]. The primary active compound present in CBE is transcinnamaldehyde, which is a powerful phenolic compound on its own, but it also contains eugenol and benzoic acid which contribute additional antimicrobial activity [10-12]. CBE has been found to effectively inhibit various foodborne pathogens, but its low aqueous solubility and high volatility make its delivery to microbial cell sites in aqueous media challenging $[9,13,14]$. Furthermore, CBE has been approved as a generally recognized as safe material for food use based on 21 Code of Federal Regulation part 172.515 [15]. The goal of this study was to develop an effective method to synthesize PNIPAAM nanoparticles with entrapped CBE to improve its delivery to foodborne pathogens and control its release with temperature stimuli. 

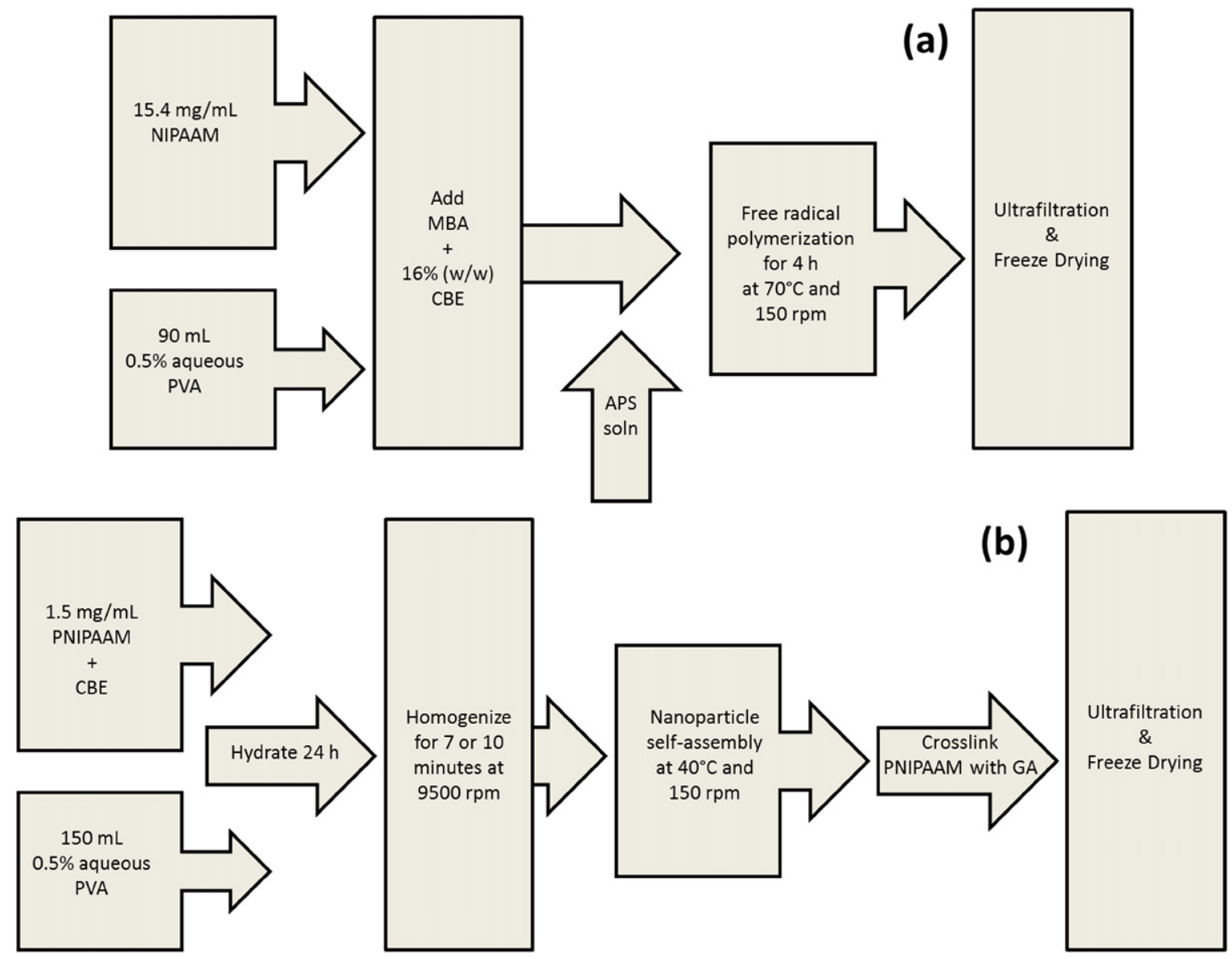

Figure 1. Schematic diagram of (a) bottom-up PNIPAAM nanoparticle synthesis by free radical polymerization and (b) top-down PNIPAAM nanoparticle synthesis method by self-assembly.

\section{Materials and methods}

\subsection{Materials}

N-isopropylacrylamide (NIPAAM) was purchased from TCI America (Portland, OR). Poly (vinyl alcohol) (PVA) (87\%, $\left.\mathrm{M}_{\mathrm{w}} \quad 30-70 \mathrm{kDa}\right), \mathrm{N}, \mathrm{N}-$ methylene-bisacrylamide (MBA), glutaraldehyde (25\%), and CBE (99\%) were purchased from Sigma Aldrich (St. Louis, MO). $\mathrm{N}, \mathrm{N}, \mathrm{N}^{\prime}, \mathrm{N}^{\prime}$-Tetramethyl-ethylenediamine (TEMED) was purchased from Alfa Aesar (Ward Hill, MA). Ammonium Persulfate (APS) was purchased from BDH Chemicals (London, England). All other reagents were of analytical grade.

\subsection{Particle synthesis}

2.2.1. Bottom-up synthesis. The bottom-up method (figure 1) used to synthesize particles was similar to the methods outlined by [16] with slight modifications. This method employs a free radical polymerization reaction to form PNIPAAM nanoparticles. Briefly, $1.54 \mathrm{~g}$ of NIPAAM monomer was dissolved in $90 \mathrm{~mL}$ of a $0.5 \%(\mathrm{w} / \mathrm{v})$ aqueous PVA solution. Once the monomer is 
dissolved, $26.2 \mathrm{mg}$ of MBA along with CBE (16\% w/w, relative to NIPAAM) was added to the reaction flask and stirred continuously with a magnetic stirrer for $30 \mathrm{~min}$. Meanwhile, the initiator solution is prepared by dissolving $62.40 \mathrm{mg}$ of APS into $10 \mathrm{~mL}$ of $0.5 \%(\mathrm{w} / \mathrm{v})$ aqueous PVA solution. Once the initial solution has mixed for $30 \mathrm{~min}$, the initiator solution is added and shaken for $4 \mathrm{~h}$ at $70^{\circ} \mathrm{C}$ in a shaking water bath (VWR International, Radnor, PA) set at $150 \mathrm{rpm}$.

After synthesis, the nanoparticles were purified by ultrafiltration to remove excess PVA, NIPAAM monomer, and non-encapsulated CBE. A Millipore-Labscale ${ }^{\mathrm{TM}}$ TFF system fitted with a $50 \mathrm{kDa}$ molecular weight cutoff Pellicon XL-Millipore (Millipore, Kankakee, IL) was used. The nanoparticles were ultrafiltered with $300 \mathrm{~mL}$ of water and $100 \mathrm{~mL}$ of the retentate was collected. Inlet pressure was $25 \mathrm{psi}$ and outlet pressure in the system was approximately 5-10 psi. After ultrafiltration, nanoparticles were kept at $-20^{\circ} \mathrm{C}$ overnight then lyophilized at $-50{ }^{\circ} \mathrm{C}$ and $1.45 \times 10^{-4}$ psi vacuum for $24 \mathrm{~h}$ in a Labconco Freeze Dry-5 unit (Labconco, Kansas City, MO). Unloaded (control) particles were synthesized through the same procedure without the addition of $\mathrm{CBE}$ into the reaction flask. Dried nanoparticles were stored at $-20^{\circ} \mathrm{C}$ until they were needed for analysis.

2.2.2. Top-down synthesis. The top-down synthesis method (figure 1) utilizes the IPN method outlined by Zhang et al $[17,18]$ with minor adjustments to develop nanoparticles. NIPAAM monomer is dissolved in distilled water to obtain a concentration of $6.7 \%(\mathrm{w} / \mathrm{w})$ and then $2.0 \%$ (w/w, relative to NIPAAM) MBA is added. Free radical polymerization was carried out in a glass flask at room temperature for $3 \mathrm{~h}$, using $1.0 \%(\mathrm{w} / \mathrm{w})$ APS and TEMED as redox initiators. After polymerization, the crosslinked hydrogel was immersed in fresh distilled water at room temperature for $48 \mathrm{~h}$ to allow all unused reactants to leach out of the gel. The water was replaced with fresh water every several hours. The final hydrogel was cut into small pieces and dried in a vacuum oven (Squared Lab Line Instruments, Melrose Park, IL) at room temperature and a pressure $\leqslant 13.3 \mathrm{kPa}$ until all moisture was removed (approximately $24 \mathrm{~h}$ ).

To form nanoparticles, $1.5 \mathrm{mg} \mathrm{mL}^{-1}$ of dried PNIPAAM was suspended in $150 \mathrm{~mL}$ of $0.5 \%(w / v)$ aqueous PVA solution with $16 \%$ (w/w) CBE or without CBE (for control particles) and allowed to hydrate overnight. Following polymer hydration, the solution was homogenized using an Ultra-Turrax T25 basic Ika (Works, Wilmington, NC) at $9500 \mathrm{rpm}$ for either $7 \mathrm{~min}$ or 10 min to break the polymer into smaller particles. Two different homogenization times were tested to determine if a longer homogenization time would significantly decrease the final nanoparticle diameter, as synthesis in the absence of homogenization yielded extremely large agglomerations of polymer $[19,20]$. Once the solutions were homogenized, they were placed in a shaking water bath (VWR International, Radnor, PA) at $40^{\circ} \mathrm{C}$ and $150 \mathrm{rpm}$ for $24 \mathrm{~h}$ to allow self-assembly into micelles. The micelles were then crosslinked with glutaraldehyde $(2: 1$ molar ratio of glutaraldehyde to monomers) to stabilize the particles. The finished particles were then purified via ultrafiltration similarly to the bottom-up procedure to remove excess reactants, and lyophilized at $-50^{\circ} \mathrm{C}$ and $1.45 \times 10^{-4} \mathrm{psi}$ vacuum for $24 \mathrm{~h}$ in a Labconco Freeze Dry-5 unit (Labconco, Kansas City, MO). Dried nanoparticles were stored at $-20^{\circ} \mathrm{C}$ until they were needed for analysis.

\subsection{Particle characterization}

2.3.1. Particle size and morphology. Aqueous suspensions of each nanoparticle were analyzed for size distribution and polydispersity index (PDI) using a Delsa ${ }^{\mathrm{TM}}$ Nano C Particle Analyzer 
(Beckman Coulter, Brea, CA). Nanoparticles were dissolved in distilled water at a concentration of $30 \mathrm{mg} \mathrm{mL}^{-1}$ and sonicated at $70 \mathrm{~W}$ (Cole Parmer sonicator 8890, Vernon Hill, IL) for $15 \mathrm{~min}$ before analysis using $1 \mathrm{~cm}$ path length plastic cuvettes at scattering angle of $165^{\circ}$, with a pinhole set to $50 \mu \mathrm{m}$, and a refractive index of 1.3328 for 120 continuous accumulation times. As well as comparing the different types of nanoparticles, the $10 \mathrm{~min}$ top-down nanoparticles were also measured for particle size at different stages of synthesis (i.e.; before freeze drying and before ultrafiltration) to elucidate the impact of each step on the particle size.

Nanoparticles were suspended in distilled water and examined using a FEI Morgagni transmission electron microscope (TEM) (FEI Company, Hillsboro, OR) at the School of Veterinary Medicine and Biomedical Sciences of Texas A\&M University (College Station, TX). Aqueous suspensions of particles were placed on 300 mesh copper grids and stained with a 2\% (w/v) uranyl acetate aqueous stain (Electron Microscopy Sciences, Hatfield, PA) to provide contrast under magnification. Excess liquid on the mesh was removed with filter paper and the grid was allowed to dry before viewing under 44000-71 000 times magnification. Observations were performed at $80 \mathrm{kV}$.

2.3.2. Entrapment efficiency (EE) and drug loading (DL). The EE and DL of CBE were measured indirectly by determining the amount of $\mathrm{CBE}$ that was present in the permeate collected during ultrafiltration. It was assumed that any CBE left in the retentate portion of the nanoparticle suspension was entrapped within particles. The amount of CBE present in the permeate was measured spectrophotometrically at $280 \mathrm{~nm}$ (Shimadzu UV-1601 spectrophotometer, Columbia, MA) in a $1 \mathrm{~cm}$ path length quartz cuvette. The EE and DL were calculated according to equations (1) and (2), respectively [21, 22]:

$$
\begin{aligned}
& \mathrm{EE}=\frac{\text { amount of active compound entrapped }}{\text { initial active compound amount }} \times 100, \\
& \mathrm{DL}=\frac{\text { amount of active compound entrapped }}{\text { amount of particles produced }} \times 100 .
\end{aligned}
$$

2.3.3. Cloud point and LCST. The LCST behavior of the PNIPAAM hydrogel was determined using differential scanning calorimetry in a Pyris 6 Perkin Elmer instrument (Pyris 5.0 Software, Boston, MA). Hydrogel samples were submerged in water and allowed to swell to equilibrium before DSC measurements were taken. Approximately $10 \mathrm{mg}$ of swollen PNIPAAM was placed into $20 \mu \mathrm{L}$ aluminum pans and sealed with one hole in their lids and scanned from $25^{\circ} \mathrm{C}$ to $50{ }^{\circ} \mathrm{C}$ at a rate of $3{ }^{\circ} \mathrm{C} \mathrm{min}^{-1}$ under nitrogen atmosphere [17].

The cloud point method [23] was used to find the thermal transition temperature of the hydrated nanoparticles. A $0.1 \% \mathrm{wt} / \mathrm{wt}$ aqueous solution of particles was prepared for each type of nanoparticle and $200 \mu \mathrm{L}$ of each particle suspension was placed into three wells of a 96-well plate. Turbidity measurements were measured at $450 \mathrm{~nm}$ using a 96 -well plate reader equipped with temperature control (VERSAmax Tunable Microplate Reader, Molecular Devices, Sunnyvale, CA). The cloud point was defined as the inflection point on a plot of absorbance versus temperature for each nanoparticle suspension as the temperature was increased from $25^{\circ} \mathrm{C}$ to $50{ }^{\circ} \mathrm{C}$ at a rate of $2{ }^{\circ} \mathrm{C}$ every $10 \mathrm{~min}$. The cloud point of the crosslinked PNIPAAM hydrogel produced for the top-down method was also measured to ensure the cloud point method and DSC method produced comparable results. 
2.3.4. Controlled release. Controlled release experiments were conducted at $25^{\circ} \mathrm{C}, 35^{\circ} \mathrm{C}$, and $45^{\circ} \mathrm{C}$ to determine the rate of CBE release below, above, or near the LCST of the nanoparticles. Nanoparticles were suspended in the release medium to achieve a $1.0 \mathrm{mg} \mathrm{mL}^{-1}$ concentration of particles (sink conditions). The release medium consisted of $10 \mathrm{~mL}$ of phosphate buffered saline (PBS, 0.15 M, pH 7.4) placed in $15 \mathrm{~mL}$ conical tubes. The tubes were placed in a shaking water bath (VWR International, Radnor, PA) set at $100 \mathrm{rpm}$ and the desired temperature, from which $1 \mathrm{~mL}$ samples were removed and analyzed for CBE content at predetermined time points up to 5 d. Additional PBS was not added after sample removal, so that the same sink conditions were maintained throughout the experiment. Samples were centrifuged at $23506 \mathrm{~g}$ for $15 \mathrm{~min}$ to separate any encapsulate material prior to spectrophotometric analysis of the supernatant at $280 \mathrm{~nm}$.

The controlled release could best be described by a semi-empirical equation formulated by Korsmeyer et al [24] (equation (3)) that accounts for Fickian diffusion and transport due to swelling effects (termed 'non-Fickian Type II transport'):

$$
\frac{M_{t}}{M_{\infty}}=k t^{n}
$$

where $M_{t} / M_{\infty}$ is the percent of antimicrobial released at time $t(s), k$ is a rate constant $(1 / s)$, and $n$ is the diffusional exponent (unit less). The release mechanism from swelling particles deviates from the traditional Fickian model since the release is not governed by diffusion of the antimicrobial alone, but also the polymer behavior upon swelling [25].

\subsection{Minimum inhibitory and bactericidal concentration (MIC and MBC)}

2.4.1. Bacterial cultures. Salmonella enterica serovar Typhimurium LT2 and Listeria monocytogenes strain Scott A were obtained from Texas A\&M University Food Microbiology Laboratory (College Station, TX). Each pathogen was chosen as a representative of Gram-negative and Gram-positive bacteria, respectively. S. typhimurium and L. monocytogenes were resuscitated in tryptic soy broth (TSB) and tryptose phosphate broth (TPB) (Becton, Dickinson and Co., Sparks, MD), respectively, by two identical consecutive transfers and incubated for $24 \mathrm{~h}$ aerobically at $35^{\circ} \mathrm{C}$. The bacterial cultures were maintained on Tryptic Soy Agar (TSA) and TSAYE (TSA containing 0.6\% (w/v) yeast extract) slants stored at $4{ }^{\circ} \mathrm{C}$ for no more than 3 months for $S$. typhimurium and L. monocytogenes, respectively. Transfers from slants were conducted similarly to the resuscitation method to prepare microorganisms for analysis.

2.4.2. Antimicrobial activity. Minimum inhibitory concentrations (MICs) for the PNIPAAM nanoparticles were determined using a broth dilution assay [26]. Growth curves were first performed at $35^{\circ} \mathrm{C}$ on each strain to correlate plate counts with optical density values at $630 \mathrm{~nm}$ (OD630) using an Epoch microplate spectrophotometer (BioTek® Instruments, Winooski, VT). Bacterial cultures were incubated $20-22 \mathrm{~h}$ and then prepared by serial dilution in doublestrength TSB (2x TSB) or TPB (2x TPB), as appropriate, for an initial inoculum of approximately $3.0 \log _{10} \mathrm{CFU} / \mathrm{mL}$ in each sample well with the appropriate amount of nutrient media present upon dilution. Initial inocula were enumerated via spread plating on TSA or Modified Oxford Agar (MOX) for S. typhimurium and L. monocytogenes; respectively and 
incubated for $24 \mathrm{~h}$ at $35^{\circ} \mathrm{C}$. Aliquots of $100 \mu \mathrm{L}$ of all antimicrobial solutions and solvent blanks were spread plated on TSA at the time of the experiment to ensure their sterility.

The MIC experiments were conducted in 96 well microtiter plates (sterilized $300 \mu \mathrm{l}$ capacity-MicroWell, NUNC, Thermo-Fisher Scientific, Waltham, MA). The nanoparticles were added to the microtiter plates as aqueous suspensions in concentrations ranging from $5000-25000 \mu \mathrm{g} \mathrm{mL}^{-1}$ for both pathogens. Equivalent volumes $(100 \mu \mathrm{L})$ of antimicrobial nanoparticle solution and bacterial inoculum in $2 \times$ broth were loaded into each test well. Negative controls were prepared with nanoparticle solutions and sterile $2 \times$ broth to account for baseline OD630 readings. Positive controls were also prepared containing inoculum and sterile distilled water or control nanoparticles to ensure nanoparticle encapsulate materials had no inhibitory effect on bacterial growth. Once plates were prepared, they were covered with a mylar plate sealer (Thermo Fisher Scientific), shaken gently, and OD630 of the wells was read $(0 \mathrm{~h})$. The microtiter plates were incubated $\left(24 \mathrm{~h}\right.$ at $\left.35^{\circ} \mathrm{C}\right)$ and shaken gently before OD630 readings were taken at $24 \mathrm{~h}$ to observe bacterial growth and inhibition over the course of the typical bacterial growth cycle. Antimicrobial test wells that showed $\leqslant 0.05$ change in OD630 after $24 \mathrm{~h}$ of incubation were considered 'inhibited' by the antimicrobial (after appropriate baseline adjustments) for that time period. The MIC for each nanoparticle and pathogen was determined by the lowest concentration of antimicrobial that inhibited growth for all test replicates [26].

All wells that showed inhibition of the test microorganism after $24 \mathrm{~h}$ were then tested for bactericidal activity by spreading $100 \mu \mathrm{L}$ from each well showing inhibition onto TSA and MOX plates for $S$. Typhimurium and L. monocytogenes; respectively, and incubating for $24 \mathrm{~h}$ at $35^{\circ} \mathrm{C}$. If no colonies were observed on the plate surfaces following incubation, the treatment concentration was considered bactericidal. The lowest concentration of nanoparticles demonstrating bactericidal activity across all replicates was considered the MBC.

\subsection{Statistical analysis}

This experiment was based on a completely randomized design with equal replications. All determinations were made in triplicate as independent experiments. All statistical analyses were performed using JMP v. 9 Software (SAS Institute, Cary, NC). Differences between variables were tested for significance using one-way analysis of variance (ANOVA) and significantly different means $(P<0.05)$ were separated using Tukey's honestly significant differences test. Controlled release data were fit to model data using JMP software and the nonlinear modeling procedure to determine rate constants $(k)$ and diffusional coefficients $(n)$. The model constants were analyzed for goodness of fit using the nonlinear procedure to determine coefficients of determination $\left(R^{2}\right)$.

\section{Results and discussion}

\subsection{Particle characterization}

3.1.1. Particle size and morphology. The nanoparticles produced (monomer (bottom up), $7 \mathrm{~min}$ and $10 \mathrm{~min}$ (top-down) were measured for particle size with and without CBE entrapped at $25^{\circ} \mathrm{C}$ and $50^{\circ} \mathrm{C}$ to determine their average diameter and polydispersity distribution (PDI) in water (table 1 and figure 2). The average diameter of all particles and their controls were smaller 
Table 1. Polydispersity (PDI) values of PNIPAAM control (unloaded) and CBE loaded nanoparticles made by the top-down (7 and $10 \mathrm{~min}$ ) and bottom-up (monomer) processes at temperatures of $25^{\circ} \mathrm{C}$ and $40^{\circ} \mathrm{C}$.

\begin{tabular}{lcc}
\hline & \multicolumn{2}{c}{ PDI } \\
\cline { 2 - 3 } Nanoparticle & $25{ }^{\circ} \mathrm{C}$ & $40{ }^{\circ} \mathrm{C}$ \\
\hline 7 min control & ${ }_{\mathrm{w}} 0.32 \mathrm{a} \pm 0.02$ & ${ }_{\mathrm{w}} 0.32 \mathrm{a} \pm 0.01$ \\
7 min CBE & ${ }_{\mathrm{w}} 0.31 \mathrm{a} \pm 0.03$ & ${ }_{\mathrm{w}} 0.23 \mathrm{a} \pm 0.11$ \\
10 min control & ${ }_{\mathrm{w}} 0.29 \mathrm{a} \pm 0.02$ & ${ }_{\mathrm{w}} 0.19 \mathrm{a} \pm 0.07$ \\
10 min CBE & ${ }_{\mathrm{w}} 0.30 \mathrm{a} \pm 0.03$ & ${ }_{\mathrm{w}} 0.26 \mathrm{a} \pm 0.07$ \\
Monomer control & ${ }_{\mathrm{w}} 1.21 \mathrm{a} \pm 0.90$ & ${ }_{\mathrm{w}} 0.38 \mathrm{a} \pm 0.09$ \\
Monomer CBE & ${ }_{\mathrm{w}} 0.25 \mathrm{a} \pm 0.02$ & ${ }_{\mathrm{w}} 0.19 \mathrm{a} \pm 0.07$ \\
\hline
\end{tabular}

${ }^{a}$ Different superscript letters within a column represent significantly different values $(P<0.05)$. ${ }_{\mathrm{w}}$ Means within a row, of the same parameter, that are not preceded by a common subscript letter are significantly different $(P<0.05)$. Values given are averages of three replicates \pm standard deviations.

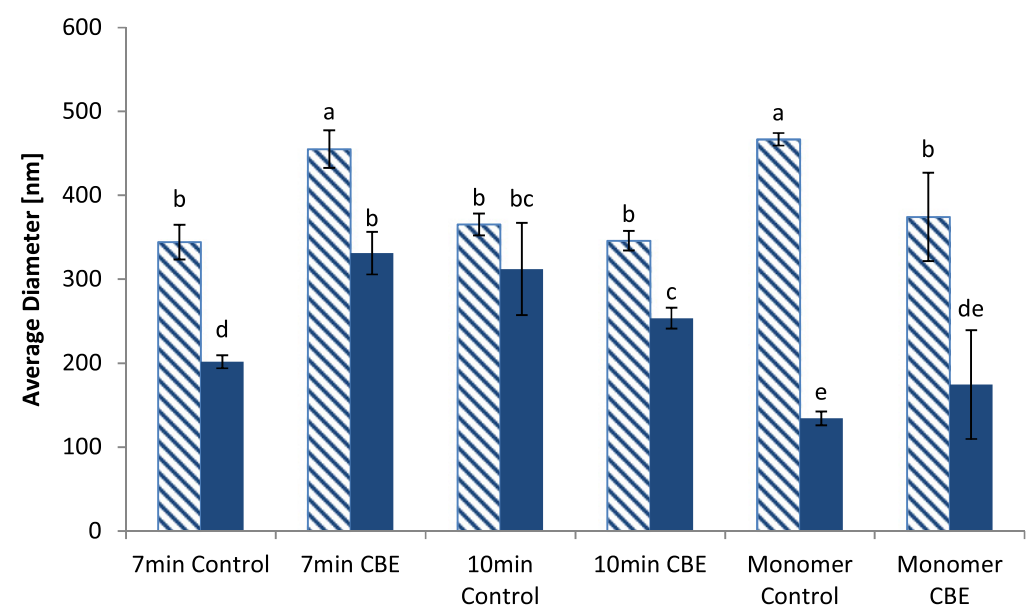

Figure 2. Average diameter of PNIPAAM nanoparticles and their controls suspended in water, synthesized through different methods at $25^{\circ} \mathrm{C}$ (pattern bars) and $40^{\circ} \mathrm{C}$ (solid bars). Monomer are nanoparticles produced by the bottom-up process and $7 \mathrm{~min}$ and $10 \mathrm{~min}$ are nanoparticles produced by the top-down process with different homogenization times. Average diameter columns that display different letters above their error bars represent significantly different values across all treatments $(P<0.05)$. Values given are averages of three replicates \pm standard deviations.

$(P<0.05)$ when heated above the LCST because the polymer contracts at the transition temperature and becomes hydrophobic [1]. The monomer control particles (bottom-up process) showed the most dramatic decrease in diameter when heated above the LCST $(466.76 \mathrm{~nm}$ compared to $134.25 \mathrm{~nm}$ ), likely because the monomer nanoparticles were made up of smaller molecules and therefore able to collapse into a more compact particle without the presence of the CBE to prevent complete collapse. The nanoparticles ranged in diameter from $344.40 \mathrm{~nm}$ to $466.76 \mathrm{~nm}$ at room temperature, with the $7 \mathrm{~min}$ control being the smallest $(P<0.05)$ and the monomer control being the largest $(P<0.05)$ particles. Once heated to $40{ }^{\circ} \mathrm{C}$, the particle 


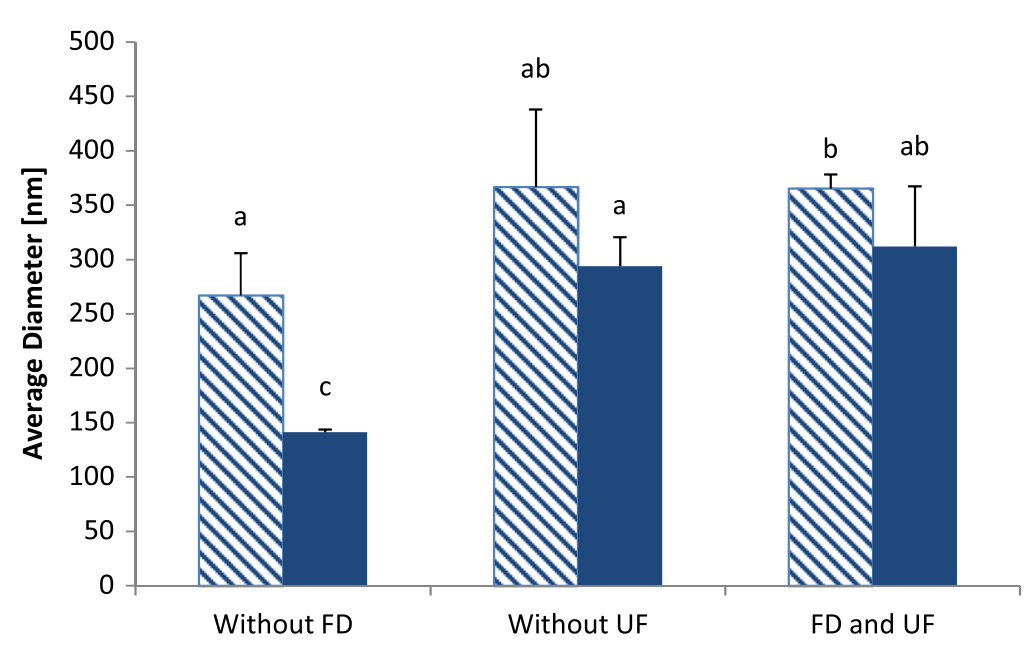

Figure 3. Comparison of average particle diameter for top-down, 10 min homogenized PNIPAAM nanoparticles at various stages of the synthesis process below $\left(25^{\circ} \mathrm{C}\right.$, pattern bars) and above $\left(40^{\circ} \mathrm{C}\right.$, solid bars $)$ the LCST. FD = Freeze drying; UF= Ultrafiltration. Average diameter columns that display different letters above their error bars represent significantly different values across all treatments $(P<0.05)$. Values given are averages of three replicates \pm standard deviations.

diameters ranged from $134.25 \mathrm{~nm}$ for the monomer control to $331.75 \mathrm{~nm}$ for the $7 \mathrm{~min}$ CBE. The top-down particles $(7$ and $10 \mathrm{~min})$ both had higher $(P<0.05)$ entrapment efficiencies, so this is likely the reason their diameters were larger $(P<0.05)$ after the particles collapsed at the LCST. The amount of time the PNIPAAM solution was homogenized did significantly impact the final particle size, with the longer homogenization time resulting in smaller $(P<0.05)$ particles.

Not only were the particle diameters of all the different nanoparticles measured, but also the $10 \mathrm{~min}$ top-down control (unloaded) particles were analyzed at different stages of the synthesis process. Figure 3 shows the average particle size without freeze drying, without ultrafiltration, and when all stages of the synthesis process have been completed. The freeze drying process seems to have the biggest impact on particle size, as the particles are significantly smaller $(P<0.05)$ when they are not lyophilized. This phenomenon is not surprising, as it has been found to occur in other studies of nanoparticle synthesis and addition of cryoprotectants such as trehalose, is sometimes recommended to minimize this effect $[21,27]$. The ultrafiltration step had little impact on the average particle diameter $(P>0.05)$, which was expected, as the goal is to remove smaller molecular weight components from the particle suspension.

The nanoparticles were similar in appearance in TEM images (figure 4) as slightly amorphous shapes, with the monomer nanoparticles (bottom-up) showing a more spherical shape than either of the top-down synthesized particles. The more amorphous shape of the topdown particles is due to the irregular polymer molecules generated by the homogenization step of the process. Other studies utilizing the bottom-up synthesis procedure displayed similarly spherical particle images [16, 28]. Wadajkar et al [16] used a similar bottom-up synthesis procedure and produced nanoparticles of a slightly smaller size and with less polydispersity, however these authors used sodium dodecyl sulfate (SDS) as a surfactant instead of PVA, which could have led to this difference. PVA was used in this study because it was found to 

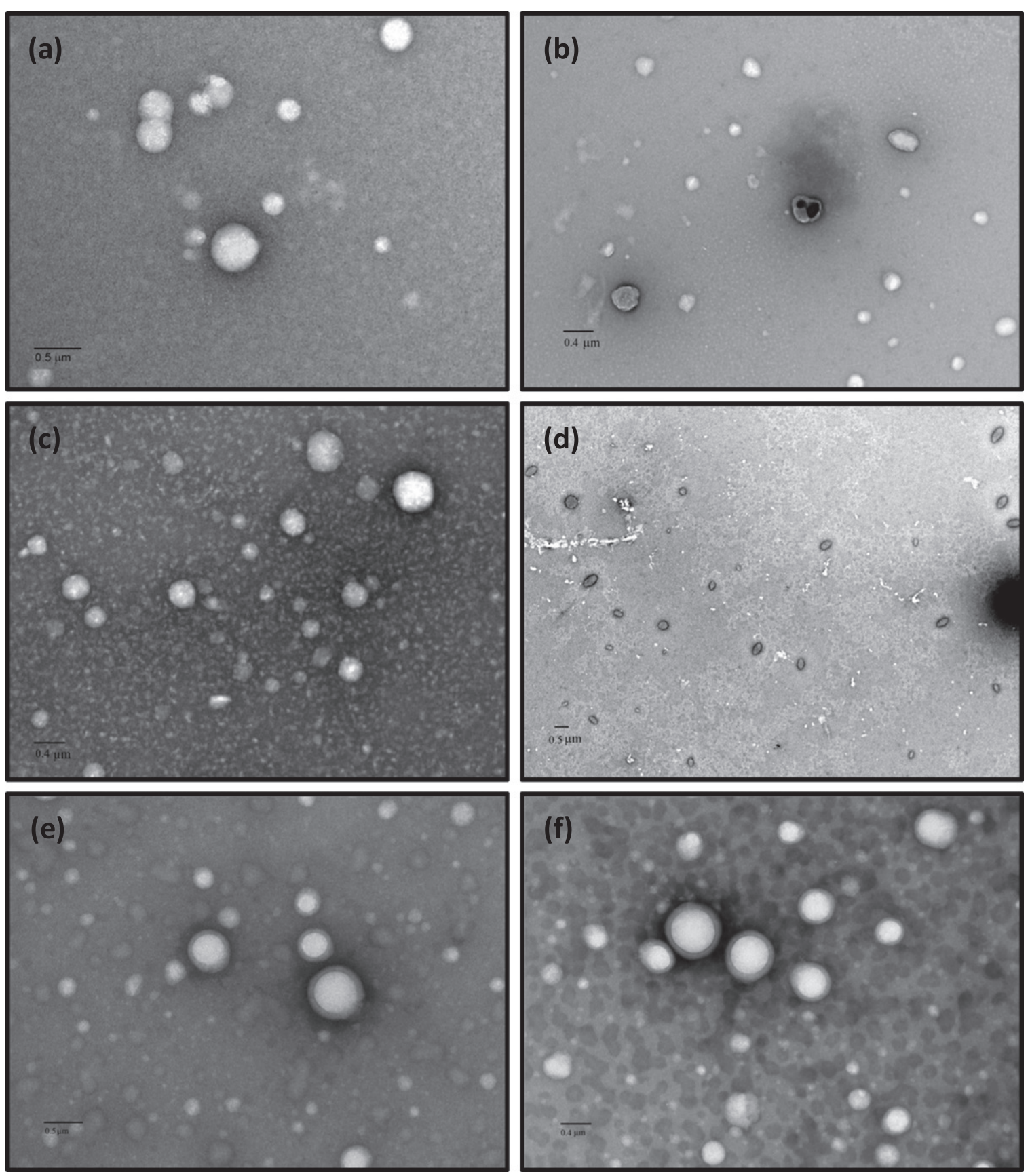

Figure 4. TEM images of PNIPAAM nanoparticles manufactured through different methods. (a) 10 min top-down control (unloaded); (b) 10 min top-down CBE; (c) 7 min top-down control; (d) 7 min top-down CBE; (e) monomer bottom-up control; (f) monomer bottom-up CBE. Observations were performed at $80 \mathrm{kV}$ using magnifications ranging from 44000 to $71000 x$. PNIPAAM nanoparticles are represented by white spherical shapes. 
Table 2. Entrapment efficiency values measured for cinnamon bark extract (CBE) in PNIPAAM nanoparticles by spectrophotometry at $280 \mathrm{~nm}$.

\begin{tabular}{lcc}
\hline Nanoparticle & Average CBE entrapped (\%) & Drug loading (\%) \\
\hline 7 min CBE & $90.24^{\mathrm{a}} \pm 0.72$ & $2.31^{\mathrm{b}} \pm 0.02$ \\
10 min CBE & $91.46^{\mathrm{a}} \pm 0.49$ & $2.34^{\mathrm{b}} \pm 0.01$ \\
Monomer CBE & $86.78^{\mathrm{a}} \pm 7.76$ & $8.99^{\mathrm{a}} \pm 0.80$ \\
\hline
\end{tabular}

Values given are averages of three replicates \pm standard deviations. Different superscript letters within a column represent significantly different values $(P<0.05)$.

produce particles with higher entrapment of CBE in preliminary testing, in comparison with particles produced with SDS as a surfactant. The bottom-up nanoparticles produced by Ramanan et al [28] were very similar in size and size distribution to the particles produced in this study. The particle size of all particles appears slightly smaller in the TEM images than the sizes measured by laser light diffraction in the particle size analyzer, but due to the high level of polydispersity in the particle samples, there could simply be a wide range of particle sizes present in the samples. Fan et al [6] displayed similar TEM images of slightly spherical, but irregular shapes for PNIPAAM nanoparticles synthesized using a top-down procedure. This author also showed an increase in particle size when PNIPAAM nanoparticles were loaded with hydrophobic material, versus unloaded control particles [6]. Amorphous nanoparticles of a similar size prepared via a top-down synthesis method were also reported by Chuang et al [7], although this study utilized a PNIPAAM-co-polymer rather than pure PNIPAAM polymer. The synthesis and analysis methods were similar, leading to relevant comparisons of the results.

3.1.2. EE and $D L$. The EE for all the nanoparticles (table 2) was similar and ranged from $86.78 \%$ for monomer particles (bottom-up process) to $91.46 \%$ for $10 \mathrm{~min}$ particles (top-down process). A high level of entrapment was achieved regardless of the synthesis method, showing that the PNIPAAM provided a highly compatible form of encapsulation for hydrophobic material. PNIPAAM possesses both hydrophilic and hydrophobic groups in the polymer chain that lead to its LCST behavior [2,29]. The nanoparticles are loaded at a temperature above their LCST, so that the hydrophobic regions collapse around the hydrophobic CBE to minimize unfavorable aqueous interactions. A similarly high EE (73.7\%) and DL (8.4\%) of hydrophobic molecules within PNIPAAM nanoparticles were found by Fan et al [6].

Similar to the EE results, the 7 and 10 min top-down PNIPAAM nanoparticles presented slightly higher DL values than the PNIPAAM nanoparticles synthesized by the bottom-up method. A DL of $100 \% \mathrm{CBE}$ entrapment would be $2.56 \%$ for the top-down nanoparticles and $10.36 \%$ for the bottom-up particles, meaning the DL values for each of the particles were close to their maximum values.

3.1.3. Cloud point and LCST. The LCST for the PNIPAAM hydrogel fell within the typical range $\left(30-35^{\circ} \mathrm{C}\right)$ for PNIPAAM polymers at $33.9^{\circ} \mathrm{C}$ via DSC determination [1]. The LCST determined by the cloud point method was slightly higher at $34.8^{\circ} \mathrm{C}$ due to the slightly lower sensitivity of this method (table 3 ). It was necessary to use the cloud point method for the nanoparticle LCST determination because the particles were protected by a matrix of PVA that prevented high enough concentrations of particles to be placed in the DSC pans. The cloud method allowed for larger volumes of particle suspensions, resulting in more reliable and 
Table 3. Lower critical solution temperatures (LCSTs) of PNIPAAM hydrogel and nanoparticles determined by differential scanning calorimetry (DSC) and the cloud point method.

\begin{tabular}{lcl}
\hline Material & Cloud point $\left({ }^{\circ} \mathrm{C}\right)$ & LCST $\left({ }^{\circ} \mathrm{C}\right)$ \\
\hline PNIPAAM polymer & $\mathrm{w} 34.8 \mathrm{a} \pm 0.1$ & $\mathrm{w} 33.9$ \\
Monomer control nanoparticle (bottom-up) & $33.5^{\mathrm{b}} \pm 0.1$ & $\mathrm{nd}$ \\
10 min control nanoparticle (top-down) & $35.4 \mathrm{a} \pm 0.1$ & $\mathrm{nd}$ \\
7 min control nanoparticle (top-down) & $35.4 \mathrm{a} \pm 0.1$ & $\mathrm{nd}$ \\
\hline
\end{tabular}

Values given are averages of three replicates \pm standard deviations.

${ }^{a}$ Means within a column that are not followed by a common superscript letter are significantly different $(P<0.05)$

${ }_{w}$ Means within a row, of the same parameter, that are not preceded by a common subscript letter are significantly different $(P<0.05)$.

nd-not determined.

consistent LCST measurements. The monomer particles had the lowest LCST at $33.5^{\circ} \mathrm{C}$, while the $7 \mathrm{~min}$ and $10 \mathrm{~min}$ nanoparticles had the same LCST values at $35.4{ }^{\circ} \mathrm{C}$. The cloud point method and DSC methods both reveal the transition temperature through different data collection.

The DSC analysis determines the endothermic transition peak caused by the heat required to break hydrogen bonds between water and the polymer, which occurs at the transition temperature. Meanwhile, the cloud point method utilizes spectrophotometric analysis to visualize the clouding of the polymer solution caused by the precipitation of the PNIPAAM at the transition temperature. Eeckman et al [30] also measured both the cloud point and the LCST value of PNIPAAM polymer, and found the values of the transition temperature to be similar regardless of the method used to measure it and close to the values reported for the nanoparticles in this study. Other studies even use the term LCST when the transition temperature has been measured by the cloud point method, likely because both methods provide similar results $[20,23]$.

3.1.4. Controlled release. The three temperatures used for the controlled release experiments were chosen as representative temperatures for the behavior of nanoparticles at temperatures above, below, or near the LCST of the particles. All of the nanoparticles showed an initial burst release followed by a slower more gradual release (figure 5). The initial burst was highest for the top-down synthesized nanoparticles at higher temperatures $\left(35^{\circ} \mathrm{C}\right.$ and $\left.40{ }^{\circ} \mathrm{C}\right)$. Above the LCST, the polymer nanoparticles exhibit a 'squeeze out' of the active compound due to the collapse of the polymer matrix resulting in the larger initial burst of release at these temperatures [2]. Release after the polymer matrix has collapsed is governed primarily by diffusion through the tighter polymer matrix [1,31]. The CBE release from the monomer particles (bottom-up process) was extremely slow, especially at $25^{\circ} \mathrm{C}$, where the concentration was too low to be measured over the course of the release study (data not shown). A study by Fan et al [6] also found a larger burst release at temperatures above the LCST of the nanoparticles. All of the nanoparticles showed a low percentage of release after $5 \mathrm{~d}$, indicating that the release would slowly continue over an extended period of time. This could potentially be beneficial for inhibiting foodborne pathogens over the course of the entire product shelf life, 


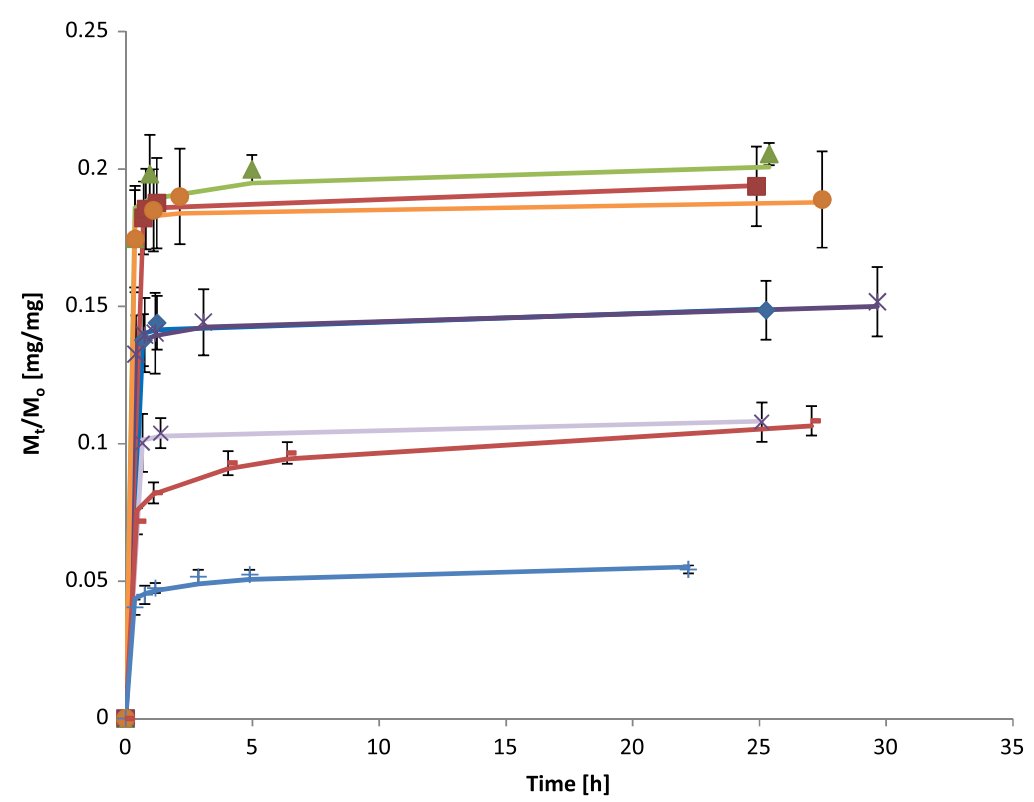

Figure 5. Controlled release profiles of PNIPAAM nanoparticles at different temperatures in $0.15 \mathrm{M}$ phosphate buffered saline (PBS, $\mathrm{pH} 7.4$ ) fit to model release equation (solid lines). ( $\diamond) 10 \mathrm{~min}$ top-down at $25^{\circ} \mathrm{C}$; ( $) 10 \mathrm{~min}$ top-down at $35^{\circ} \mathrm{C},(\Delta)$ 10 min top-down at $40^{\circ} \mathrm{C},(X) 7$ min top-down at $25^{\circ} \mathrm{C}$; (X) 7 min top-down at $35^{\circ} \mathrm{C}$; (•) 7 min top-down at $40^{\circ} \mathrm{C},(-)$ monomer bottom-up at $35^{\circ} \mathrm{C} ;(+)$ monomer bottomup at $40^{\circ} \mathrm{C}$. Symbols are means of three replicate measurements and error bars represent standard deviations.

for example fresh and fresh-cut produce, which have a shelf life of 14-21 d. Zhang et al [17] also measured a slow, gradual release of active material from PNIPAAM hydrogels over the course of $96 \mathrm{~h}$; however, their analysis was on large pieces of polymer rather than nanoparticles. The polymer was crosslinked in a process similar to the polymer synthesis used for the topdown process, and the total cumulative release ranged from 20 to 40\% [17], similar to the range of release from the top-down particles synthesized in this study. The PNIPAAM nanoparticles show excellent potential for encapsulating essential oils and protecting them from degradation or losses due to volatilization. The monomer (bottom-up) nanoparticles showed similar release profiles to those synthesized by Ramanan et al [28] who found a low level of release from monomer nanoparticles with an initial burst followed by a more gradual release. This study also found a higher rate of release from particles placed in release media above the LCST than those maintained at a release temperature below the LCST [28].

The release data points from the controlled release experiments fit well to the release equation proposed for spherical, swellable polymers (equation (2)) as was expected for these nanoparticle systems (table 4). All coefficients of determination $\left(R^{2}\right)$ were greater than 0.95 and therefore were not shown in table 4 .

\subsection{Minimum inhibitory and bactericidal concentration (MIC) and (MBC)}

The MIC values (table 5) for CBE encapsulated in both of the top-down PNIPAAM nanoparticles ( 7 min $\mathrm{CBE}$ and $10 \mathrm{~min} \mathrm{CBE}$ ) was very similar and significantly lower than the MIC for CBE encapsulated in the bottom-up PNIPAAM nanoparticles (monomer CBE) against 
Table 4. Controlled release model rate coefficients $(k)$ and diffusion constants $(n)$ for PNIPAAM nanoparticles with entrapped CBE synthesized by bottom-up (monomer) and top-down processes ( 7 and $10 \mathrm{~min}$ ).

\begin{tabular}{|c|c|c|c|c|c|c|}
\hline \multirow[b]{3}{*}{ Nanoparticle } & \multicolumn{6}{|c|}{ Release temperature } \\
\hline & \multicolumn{2}{|l|}{$25^{\circ} \mathrm{C}$} & \multicolumn{2}{|c|}{$35^{\circ} \mathrm{C}$} & \multicolumn{2}{|c|}{$40^{\circ} \mathrm{C}$} \\
\hline & $k_{\mathrm{r}}\left(\mathrm{s}^{-n}\right)$ & $n_{\mathrm{r}}$ & $k_{\mathrm{r}}\left(\mathrm{s}^{-n}\right)$ & $n_{\mathrm{r}}$ & $k_{\mathrm{r}}\left(\mathrm{s}^{-n}\right)$ & $n_{\mathrm{r}}$ \\
\hline $10 \mathrm{~min}$ & $\mathrm{x} 3.77 \times 10^{-4 \mathrm{~b}} \pm 1.88 \times 10^{-5}$ & ${ }_{x} 0.59 \mathrm{a} \pm 2.51 \times 10^{-3}$ & $y^{5.85} \times 10^{-4} a \pm 3.19 \times 10^{-5}$ & $\mathrm{y} 0.61 \mathrm{a} \pm 2.48 \times 10^{-3}$ & $\mathrm{y} 6.34 \times 10^{-4 \mathrm{c}} \pm 1.95 \times 10^{-5}$ & $\mathrm{z}^{0.62^{\mathrm{c}} \pm 1.11 \times 10^{-3}}$ \\
\hline $7 \mathrm{~min}$ & $2.60 \times 10^{-4} a \pm 1.08 \times 10^{-5}$ & ${ }_{x} 0.57^{b} \pm 2.08 \times 10^{-3}$ & $5.44 \times 10^{-4} a \pm 2.92 \times 10^{-5}$ & $\mathrm{y} 0.61 \mathrm{a} \pm 2.83 \times 10^{-3}$ & $y^{5.51 \times 10^{-4 b} \pm 2.62 \times 10^{-5}}$ & $\mathrm{y} 0.61^{\mathrm{b}} \pm 5.92 \times 10^{-3}$ \\
\hline Monomer & N/A & N/A & $x^{2.33 \times 10^{-4 b} \pm 8.05 \times 10^{-6}}$ & ${ }_{x} 0.58^{b} \pm 1.72 \times 10^{-3}$ & $x^{2} .66 \times 10^{-4} a \pm 6.02 \times 10^{-6}$ & ${ }_{x} 0.58 \mathrm{a} \pm 1.09 \times 10^{-3}$ \\
\hline
\end{tabular}

Values given are averages of three replicates \pm standard deviations.

N/A-equation (3) did not fit experimental data.

${ }^{a}$ Different superscript letters within a column represent significantly different values $(P<0.05)$.

${ }_{\mathrm{w}}$ Means within a row, of the same parameter, that are not preceded by a common subscript letter are significantly different $(P<0.05)$. 
Table 5. MIC and MBC of CBE loaded PNIPAAM nanoparticles against Salmonella enterica Typhimurium LT2 and Listeria monocytogenes Scott A for CBE loaded PNIPAAM nanoparticles synthesized by bottom-up (monomer) and top-down processes (7 and $10 \mathrm{~min}$ ).

\begin{tabular}{|c|c|c|}
\hline Nanoparticle & $\mathrm{MIC}^{1}\left(\mu \mathrm{g} \mathrm{mL}^{-1}\right)$ & $\operatorname{MBC}\left(\mu \mathrm{g} \mathrm{mL}^{-1}\right)$ \\
\hline \multicolumn{3}{|c|}{ Salmonella enterica Typhimurium LT2 } \\
\hline $7 \mathrm{~min} \mathrm{CBE}$ & $345.79^{\mathrm{a}}$ & $345.79^{\mathrm{a}}$ \\
\hline 10 min CBE & $347.09^{\mathrm{b}}$ & $347.09^{\mathrm{b}}$ \\
\hline Monomer CBE & $2061.80^{c}$ & $>2061.80^{2 c}$ \\
\hline \multicolumn{3}{|c|}{ Listeria monocytogenes Scott A } \\
\hline $7 \mathrm{~min} \mathrm{CBE}$ & $461.06^{\mathrm{a}}$ & $576.32^{\mathrm{a}}$ \\
\hline 10 min $\mathrm{CBE}$ & $347.09^{\mathrm{b}}$ & $>578.48^{2 b}$ \\
\hline Monomer CBE & $1237.08^{c}$ & $>2061.80^{c}$ \\
\hline
\end{tabular}

\footnotetext{
${ }^{1}$ Values are the lowest concentration of nanoencapsulated CBE for which a $\leqslant 0.05$ OD630 change was observed after $24 \mathrm{~h}$ incubation at $35^{\circ} \mathrm{C}$ in tryptic soy broth. MIC and MBC values are given based on CBE concentration.

${ }^{2}$ Values preceded by a higher than $(>)$ means that tested concentrations were not sufficient to determine the MIC or MBC values.

${ }^{\mathrm{a}, \mathrm{b}}$ Means within a column for the same bacterium which are not followed by a common superscript letter are significantly different $(P<0.05)$.
}

both microorganisms (Salmonella and Listeria). This is likely a result of the much slower initial release from the monomer nanoparticles. The MIC experiments were conducted in a short time period $(24 \mathrm{~h})$ that did not provide enough time for the monomer nanoparticles to release as much CBE into the growth media. The 10 min CBE nanoparticles had the same MIC value against both $S$. typhimurium and L. monocytogenes, while the $7 \mathrm{~min}$ CBE nanoparticle had a slightly higher MIC against L. monocytogenes. Both the $7 \mathrm{~min}$ and $10 \mathrm{~min} \mathrm{CBE}$ nanoparticles showed bactericidal activity against the $S$. typhimurium at the same concentration as their MIC, but the concentrations tested against L. monocytogenes were not high enough to exhibit bactericidal activity. The reason for the higher MIC and MBC values for L. monocytogenes for all the nanoparticles is because Gram-positive bacteria are less susceptible to inhibition by essential oils [32]. The primary mode of action for essential oils is through the cell membrane, and the Gram-positive cell membrane is more difficult for essential oils to access $[32,33]$. The 10 min and 7 min PNIPAAM particles both had lower MIC values than free CBE against both $S$. typhimurium and L. monocytogenes found in a previous study, $400 \mu \mathrm{g} \mathrm{mL}^{-1}$ and $500 \mu \mathrm{g} \mathrm{mL}^{-1}$, respectively [13]. Similarly for the MBC values, a lower level of nanoparticles was effective against the Salmonella than the L. monocytogenes. Bactericidal activity was achieved for the $7 \mathrm{~min}$ and $10 \mathrm{~min}$ (top-down) nanoparticles for Salmonella, but the concentration of monomer (bottom-up) nanoparticles tested, was not sufficiently high to kill all the bacterial cells. Only the $7 \mathrm{~min}$ (top-down) nanoparticles exhibited bactericidal activity at the concentrations tested against L. monocytogenes, as it is less susceptible to the antimicrobial activity of essential oils as previously mentioned.

The hydrophobic nature of CBE makes its delivery to pathogen cites challenging in a aqueous environment. Nanoencapsulation in PNIPAAM improves the aqueous solubility of the 
essential oil and consequently, improves its delivery to microbial cells in aqueous media [6,34]. Once the essential oil is delivered to the pathogen cell, its hydrophobic character is advantageous in helping the antimicrobial material partition into the cell membrane $[6,12]$. Without the protective PNIPAAM encapsulation, the CBE may have a tendency to coalesce is aqueous solution in order to minimize unfavorable hydrophobic interactions or even be lost to the atmosphere by volatilization [35]. In the case of the monomer (bottom-up) PNIPAAM particles, the encapsulation may in fact be too protective, thereby limiting the amount of CBE that is released initially. This resulted in the higher MIC values against the pathogens because not enough CBE was available to inhibit the cell growth. The increase in temperature above the LCST for the top-down particles caused a contraction of the polymer matrix that quickly forced out some of the entrapped CBE. This contraction created a larger 'burst' of CBE release initially than the bottom-up nanoparticles. This helped inhibit the bacteria by quickly weakening the bacteria cells by delivering a large initial dose of antimicrobial to the pathogen sites, just as the environmental temperature is reaching the optimal range for pathogen growth $\left(35^{\circ} \mathrm{C}\right)$.

\section{Conclusions}

Different processes of synthesis were compared for the production of temperature-responsive PNIPAAM particles for controlled release of CBE. The goal was to determine the best method to synthesize particles to be used for antimicrobial controlled delivery, as no previous research has been conducted related to the use of PNIPAAM in this application. The top-down versus bottom-up synthesis methods yielded particles with significantly different characteristics, especially their release profiles and antimicrobial activities. The PNIPAAM particles synthesized via the top-down procedure had a much faster release, which led to a greater antimicrobial activity. Both of the top-down nanoparticles performed similarly, so the $7 \mathrm{~min}$ homogenization time nanoparticles would be the best for this application, as the process time is shorter and little improvement was seen by using a slightly longer homogenization. This synthesis procedure also has more promise for use in the food industry because it allows for much more effective purification steps to remove potentially harmful monomers and reagents. The particles produced through the bottom-up procedure could be advantageous in applications were a robust encapsulation was necessary to protect a hydrophobic active compound over a longer period of time than what is needed for antimicrobial delivery.

\section{Acknowledgements}

The authors would like to acknowledge Dr Taylor, Animal Science Department, Texas A\&M University (TAMU) for technical assistance with MIC and MBC tests, Drs Fernando and Nikolov, Biological and Agricultural Engineering Department, TAMU, for use of lab facilities and equipment for particle size analysis and ultrafiltration, respectively.

\section{References}

[1] Schild H 1992 Poly(N-isopropylacrylamide): experiment, theory and application Prog. Polym. Sci. 17 163-249 
[2] Gran M L 2011 Metal-Polymer Nanoparticulate Systems for Externally-Controlled Delivery [dissertation]. (Austin, TX: The University of Texas at Austin)

[3] Pelton R 2000 Temperature-sensitive aqueous microgels Adv. Colloid Interface Sci. 85 1-33

[4] Sershen S, Westcott S, Halas N and West J 2000 Temperature-sensitive polymer-nanoshell composites for photothermally modulated drug delivery J. Biomed. Mater. Res. $51293-8$

[5] Neradovic D, Soga O, Van Mostrum C and Hennink W 2004 The effect of the processing and formulation parameters on the size of nanoparticles based on block copolymers of poly(ethylene glycol) and poly $(N-$ isopropylacrylamide) with and without hydrolytically sensitive groups Biomaterials 25 2409-18

[6] Fan L et al 2008 Novel super $\mathrm{pH}$-sensitive nanoparticles responsive to tumor extracellular $\mathrm{pH}$ Carbohydr. Polym. 73 390-400

[7] Chuang C Y, Don T M and Chiu W Y 2010 Synthesis and characterization of stimuli-responsive porous/ hollow nanoparticles by self-assembly of chitosan-based graft copolymers and application in drug release J. Polym. Sci. A 48 2377-87

[8] Kalemba D and Kunicka A 2003 Antibacterial and antifungal properties of essential oils Curr. Med. Chem. 10 813-29

[9] Valero M and Salmeron M 2003 Antibacterial activity of 11 essential oils against Bacillus cereus in tyndallized carrot broth Int. J. Food Microbiol. 85 73-81

[10] Davidson P and Taylor T 2007 Chemical preservatives and natural antimicrobial compounds Food Microbiology: Fundamentals and Frontiers ed M P Doyle, L R Beuchat and T J Montville 3rd edn (Washington, DC: ASM Press) pp 520-56

[11] Burt S 2004 Essential oils: their antibacterial properties and potential applications in foods: a review Int. J. Food Microbiol. 94 223-53

[12] Amalaradjou M A and Venkitanarayanan K 2011 Effect of trans-cinnamaldehydeon inhibition and inactivation of Cronobacter sakazakii biofilm on abiotic surfaces J. Food Prot. 74 200-8

[13] Hill L E, Gomes C and Taylor M T 2013 Characterization of beta-cyclodextrin inclusion complexes containing essential oils (trans-cinnamaldehyde, eugenol, cinnamon bark, and clove bud extracts) for antimicrobial delivery applications LWT-Food Sci. Technol. 51 86-93

[14] Ayala-Zavala J, Soto-Valdez H, Gonzalez-Leon A, Alvarez-Parrilla E, Martin-Belloso O and Gonzalez-Aguilar G A 2008 Microencapsulation of cinnamon leaf (Cinnamon zeylanicum) and garlic (Allium sativum) oils in beta-cyclodextrin J. Incl. Phenom. Macrocycl. Chem. 60 359-68

[15] Code of Federal Regulations (CFR) 2009 Title 21, vol 3, Part 172.515 Food additives permitted for direct addition to food for human consumption: synthetic flavoring substances and adjuvants pp 56-63

[16] Wadajkar A S, Koppolu B, Rahimi M and Nguyen K T 2009 Cytotoxic evaluation of N-isopropylacrylamide monomers and temperature-sensitive poly(N-isopropylacrylamide) nanoparticles J. Nanopart. Res. 11 1375-82

[17] Zhang X Z, Wu D Q and Chu C C 2004 Synthesis, characterization and controlled drug release of thermosensitive IPN-PNIPAAm hydrogels Biomaterials 25 3793-805

[18] Zhang X, Wu D Q and Chu C C 2004 Synthesis and characterization of partially biodegradable, temperature and $\mathrm{pH}$ sensitive Dex-MA/PNIPAAm hydrogels Biomaterials 25 4719-30

[19] Chuang C Y, Don T M and Chiu W Y 2009 Synthesis of chitosan-based thermo- and pH-responsive porous nanoparticles by temperature-dependent self-assembly method and their application in drug release J. Polym. Sci. A 47 5126-36

[20] Zhang L, Guo R, Yang M, Jiang X and Liu B 2007 Thermo and pH dual-responsive nanoparticles for anticancer drug delivery Adv. Mater. 19 2988-92

[21] Gomes C, Moreira R G and Castell-Perez E 2011 Poly (DL-lactide-co-glycolide) (PLGA) nanoparticles with entrapped trans-cinnamaldehyde and eugenol for antimicrobial delivery applications J. Food Sci. 76 N16-24

[22] Iannitelli A et al 2011 Potential antibacterial activity of carvacrol-loaded poly(DL-lactide-co-glycolide) (PLGA) nanoparticles against microbial biofilm Int. J. Mol. Sci. 12 5039-51 
[23] Kim K H, Kim J and Jo W H 2005 Preparation of hydrogel nanoparticles by atom transfer radical polymerization of $\mathrm{N}$-isopropylacrylamide in aqueous media using PEG macro-initiator Polymer 46 2836-40

[24] Korsmeyer R W, Lustig S R and Peppas N A 1986 Solute and penetrant diffusion in swellable polymers: I. Mathematical modeling J. Polym. Sci. B 24 395-408

[25] Arifin D Y, Lee L Y and Wang C H 2006 Mathematical modeling and simulation of drug release from microspheres: implications to drug delivery systems Adv. Drug Deliv. Rev. 58 1274-325

[26] Brandt A L, Castillo A, Harris K B, Keeton J T, Hardin M D and Taylor T M 2010 Inhibition of Listeria monocytogenes by food antimicrobials applied singly and in combination J. Food Sci. 75 M557-63

[27] Astete C E and Sabliov C M 2006 Synthesis and characterization of PLGA nanoparticles J. Biomater. Sci. Polym. Ed. 17 247-89

[28] Ramanan R M K, Chellamuthu P, Tang L and Nguyen K T 2006 Development of a temperature-sensitive composite hydrogel for drug delivery applications Biotechnol. Prog. 22 118-25

[29] Philips M A, Gran M L and Peppas N A 2010 Targeted nanodelivery of drugs and diagnostics Nano Today 5 143-59

[30] Eeckman F, Amighi K and Moes A J 2001 Effect of some physiological and non-physiological compounds on the phase transition temperature of thermoresponsive polymers intended for oral controlled-drug delivery Int. J. Food Microbiology 222 259-70

[31] Ritger P L and Peppas N A 1987 A simple equaiton for description on solute release: II. Fickian and anomalous release from swellable devices J. Control. Release. 5 37-42

[32] Walsh S E, Maillard J Y, Russell A, Catrenich C, Charbonneau D and Bartolo R 2003 Activity and mechanisms of action of selected biocidal agents on Gram-positive and -negative bacteria J. Appl. Microbiology 94 240-7

[33] Raybaudi-Massilia R M, Mosqueda-Melgar J, Soliva-Fortuny R and Martin-Belloso O 2009 Control of pathogenic and spoilage microorganisms in fresh-cut fruits and fruit juices by traditional and alternative natural antimicrobials Compr. Rev. Food Sci. Food Safety 8 157-80

[34] Ravichandran M, Hettiarachchy N S, Ganesh V, Ricke S C and Singh S 2011 Enhancement of antimicrobial activities of naturally occurring phenolic compounds by nanoscale delivery against Listeria monocytogenes, Escherichia coli 0157:H7 and Salmonella typhimurium in broth and chicken meat system J. Food Safety 31 462-71

[35] Lapidot T, Walker M D and Kanner J 2002 Can apple antioxidants inhibit tummor cell proliferation? Generation of $\mathrm{H}_{2} \mathrm{O}_{2}$ during interaction of phenolic ocmpounds with cell culture media J. Agric. Food Chem. 50 3156-60 